\title{
INFLUENCE OF GRAVITATIONAL LENSING ON \\ ESTIMATES OF $\Omega$ IN NEUTRAL HYDROGEN
}

\author{
MATTHIAS BARTELMANN ${ }^{1,2}$ AND ABRAHAM LOEB ${ }^{1}$ \\ ${ }^{1}$ Harvard-Smithsonian Center for Astrophysics \\ 60 Garden Street, Cambridge, MA 02138, USA, and \\ ${ }^{2}$ Max-Planck-Institut für Astrophysik \\ Postfach 1523, 85740 Garching, Germany
}

\section{Introduction}

A wealth of observational data supports the commonly held view that damped Lyman- $\alpha$ (Ly $\alpha)$ absorption in QSO spectra is associated with neutral-hydrogen (HI) disks in spiral galaxies. Most of the HI probed by QSO absorption lines is traced by damped Ly $\alpha$ lines because of their high column densities, $N>10^{20} \mathrm{~cm}^{-2}$. The spiral galaxies hosting the HI disks can act as gravitational lenses on the QSOs. If the HI column density increases towards the center of the disks, as suggested by observations of local galaxies, the magnification bias preferentially selects for high columndensity systems. The estimates of HI in damped Ly $\alpha$ systems can then systematically be distorted by gravitational lensing.

\section{A Model for Lensing by Damped Ly $\alpha$ Absorbers}

We use a simple model to quantify this influence of lensing on the observed cosmological density parameter in HI, $\Omega_{\mathrm{HI}}$ (Bartelmann \& Loeb 1995). Its ingredients are:

- The lenses are modeled as singular isothermal spheres with a mean velocity dispersion of $\sigma_{v} \sim 160 \mathrm{~km} \mathrm{~s}^{-1}$.

- Their spatial number density is assumed to be constant in comoving coordinates.

- The HI column densities in the absorbers are modeled by exponential disks adapted to observational data.

- The disks are randomly inclined relative to the line-of-sight. 

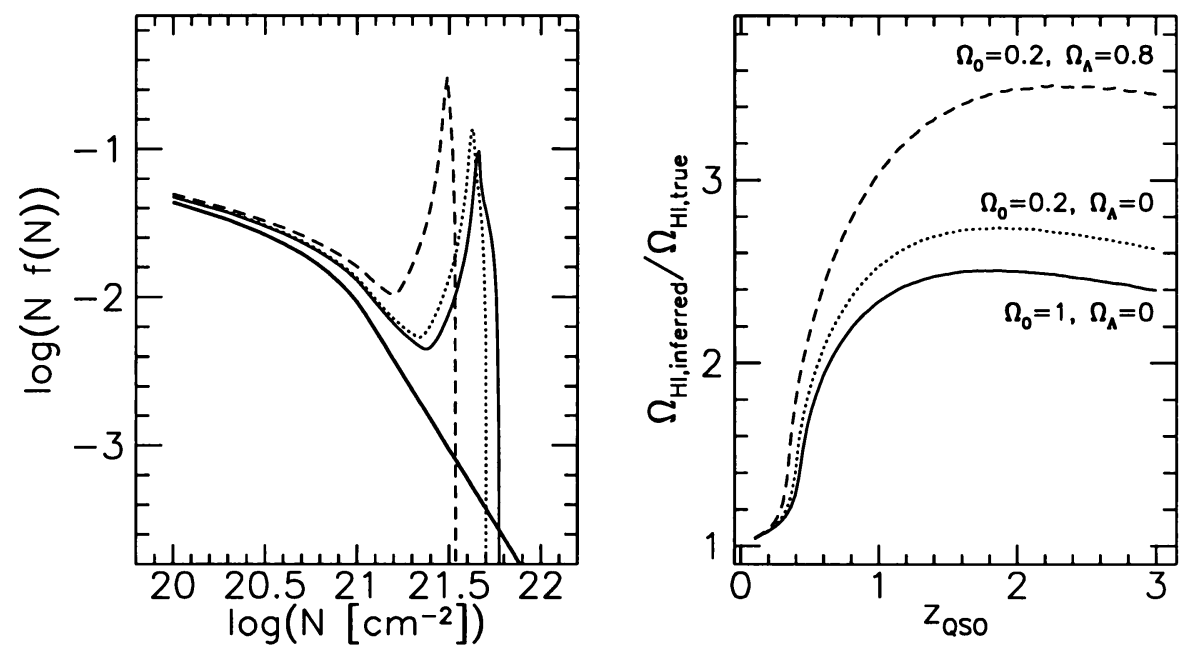

Figure 1. $f(N)$ and $\Omega_{\mathrm{HI}}$ are influenced by lensing; see the text for an explanation.

We investigate the influence of gravitational lensing on the column-density distribution, $f(N)$, of the damped Ly $\alpha$ absorption lines, and on $\Omega_{\mathrm{HI}}$. In terms of $f(N), \Omega_{\mathrm{HI}}$ is determined by $\Omega_{\mathrm{HI}} \propto \int \mathrm{d} N N f(N)$.

\section{Results}

The left frame in figure 1 shows $N f(N)$ for a QSO redshift of $z_{\mathrm{QSO}}=3$ and absorber redshifts $0.4 \leq z_{\text {abs }} \leq 1$. The heavy line shows the result neglecting lensing for comparison. The different lines represent the results for three different choices for the cosmological parameters, as indicated in the right frame. The most prominent feature in $f(N)$ is the peak followed by an abrupt cut-off. The peak arises because the magnification bias exaggerates the number of high column-density systems. The cutoff is due to the fact that the bright image of a QSO can not come arbitrarily close to the center of the absorber because light rays are bent by the lens. Although the fainter image can approach the absorber center, the equivalent width of the absorption line and therefore the inferred column density are dominated by the spectrum of the bright image. The effects on $\Omega_{\mathrm{HI}}$ are seen in the right frame, where the absorber redshift varies between zero and $z_{\mathrm{QSO}} . \Omega_{\mathrm{HI}}$ is overestimated by factors of a few, and the redshift dependence of lensing effects can produce a substantial spurious evolution of $\Omega_{\mathrm{HI}}$.

\section{References}

Bartelmann, M., \& Loeb, A., 1995, ApJ, submitted 